\title{
Variabilité hydrologique et biologique du golfe du Lion. II. Productivité sur le bord interne du courant
}

\author{
Pascal CONAN $^{a *}$, Mireille PUJO-PAY ${ }^{\text {b }}$, Patrick RAIMBAULT ${ }^{\mathrm{a}}$, Michel LEVEAU ${ }^{\mathrm{a}}$ \\ ${ }^{a}$ Centre d'océanologie de Marseille, LOB-UMR 6535, faculté des sciences de Luminy, case 901, 13288 Marseille \\ cedex 09 , France \\ * conan@ com.univ-mrs.fr \\ ${ }^{\mathrm{b}}$ Laboratoire d'océanographie biologique, UMR 7621, laboratoire Arago, BP 44, 66651 Banyuls-sur-Mer cedex, France
}

(Revisé le 17 juin 1998, accepté le 24 juin 1998)

\begin{abstract}
Hydrological and biological variability in the Gulf of Lions. II. Productivity on the inner edge of the North Mediterranean Current. An estimation of the annual productivity in the Gulf of Lions has been made in the 0-100 m layer using a Let-Go system, with monthly measurements at a fixed station. A value of 140 to $150 \mathrm{~g} \mathrm{~m}^{-2} \mathrm{a}^{-1}$ of C is found, of which around $75 \%$ occurs in the upper $50 \mathrm{~m}$. The seasonal production is described according to three distinct patterns during the two hydrological seasons: (1) variable production in winter; (2) surface bloom in late winter; (3) homogeneous and mean production in summer with a clear Deep Chlorophyll Maximum. The hydrological winter phase represented $60 \%$ of total annual production. The seasonal duality of the hydrodynamic system and the mesoscale variations of the Northern Mediterranean Current account for the variability and the evolution of the three production patterns. $\odot$ Elsevier, Paris
\end{abstract}

\section{primary production / budget / nitrate / chlorophyll / seasonal cycle}

Résumé - La productivité annuelle du golfe du Lion a été estimée dans la couche 0-100 m à l'aide d'un système Let-Go, par des mesures mensuelles à une station fixe. Une valeur de 140 à $150 \mathrm{~g} \mathrm{~m}^{-2} \mathrm{a}^{-1}$ de C est obtenue, avec une contribution de $75 \%$ pour la couche $0-50 \mathrm{~m}$. Le système productif saisonnier est décrit selon trois situations de base s'inscrivant dans deux saisons hydrologiques: (1) production variable en hiver ; (2) efflorescence algale en surface à la fin de la saison hydrologique hivernale ; (3) production homogène et moyenne durant l'été, avec présence d'un Maximum Profond de Chlorophylle. La saison hydrologique hivernale participe pour $60 \%$ au bilan annuel total de carbone. La dualité saisonnière de l'hydrodynamisme et les variations à mésoéchelle du Courant Nord Méditerranéen expliquent la variabilité et l'évolution des trois situations de base. (C) Elsevier, Paris

production primaire / bilan / nitrate / chlorophylle / cycle saisonnier

\section{INTRODUCTION}

Les régions côtières sont des sites privilégiés pour les flux de matière. Il est donc important de quantifier les variations de leur productivité et leur contribution dans le bilan global des échanges de carbone [5]. En Méditerranée nord-occidentale, le golfe du Lion a un fonctionnement hydrodynamique complexe [13] qui a des répercussions significatives sur le système biogéochimi- que de la région [30]. D'importantes variations spatiales et temporelles de sa productivité ont été décrites $[6,31]$ et ont conduit Minas [42] puis Lefèvre et al. [37] à subdiviser le golfe du Lion en quatre zones géographiques distinctes. (1) Le golfe de Marseille à l'est, dont le bilan de la production primaire s'élève à $88 \mathrm{~g} \mathrm{~m}^{-2} \mathrm{a}^{-1}$ de $\mathrm{C}$. (2) Le panache du Rhône au centre et vers l'ouest, dont le bilan de la production primaire varie entre 300 et plus de $1500 \mathrm{~g} \mathrm{~m}^{-2} \mathrm{a}^{-1}$ de $\mathrm{C}[66]$. (3) La zone de dilution rhoda- 
nienne, dont le bilan de la production primaire est compris entre 86 et $142 \mathrm{~g} \mathrm{~m}^{-2} \mathrm{a}^{-1}$ de C [31]. (4) La partie sud du golfe comprenant le Courant Nord Méditerranéen (CNM) et les zones frontales associées au CNM [59]. Le bilan de la production primaire est faible dans le courant ( $<73 \mathrm{~g} \mathrm{~m}^{-2} \mathrm{a}^{-1} \mathrm{de} \mathrm{C}$ ), mais peut atteindre des valeurs supérieures à $500 \mathrm{~g} \mathrm{~m}^{-2} \mathrm{a}^{-1} \mathrm{dc} \mathrm{C}$ dans les zones frontales.

Pour l'ensemble du golfe du Lion, Lefèvre et al. [37] proposent un bilan de la productivité de 78 à $120 \mathrm{~g} \mathrm{~m}^{-2} \mathrm{a}^{-1}$ de $\mathrm{C}$, en accord avec la valeur de $106 \mathrm{~g} \mathrm{~m}^{-2} \mathrm{a}^{-1}$ de $\mathrm{C}$ obtenue par imagerie satellitale [48]. Une tentative de bilan à méso-échelle a récemment été effectuée par modélisation de l'écosystème méditerranéen [38]. Cette approche vise à simuler les variations de la production primaire et l'exportation de matière dans le golfe du Lion, mais la généralisation est rendue difficile par le manque de données expérimentales et par l'absence de suivi saisonnier in situ de l'écosystème à une échelle suffisamment réduite.

L'objectif principal de cette étude est une description des cycles saisonniers et des échelles de variabilité du système biogéochimique à l'entrée du golfe du Lion, en relation avec l'évolution du système hydrodynamique (partie I, [13]). Des mesures mensuelles de la production primaire à une station fixe permettent une nouvelle évaluation de la productivité de la zone.

\section{MATÉRIEL ET MÉTHODES}

La zone prospectée, le matériel et les méthodes utilisés pour la récolte et l'analyse des variables physiques, chimiques et biologiques sont détaillés dans la partie I [13]. La station M3 (figure 1 partie 1, reprise figure $4 a$ ), siège des mesures de la production primaire, est localisée au centre de la radiale $\left(43^{\circ} 02,0^{\prime} \mathrm{N} / 05^{\circ} 12,5^{\prime} \mathrm{E}\right)$ dans le canyon de Marseille, sur des fonds de $1000 \mathrm{~m}$.

La profondeur de la couche euphotique (Ze) a été mesurée à l'aide d'un capteur de PAR (Photosynthetic Available Radiation) de type biospherical QSP 200L couplé à une bathysonde seabird $911+$. La profondeur du $1 \%$ de lumière oscille saisonnièrement entre 40 et $70 \mathrm{~m}$ [9]. L'épaisseur de la couche productive étant proche de $1,5 \mathrm{Ze} \mathrm{[47],} \mathrm{les} \mathrm{mesures} \mathrm{et} \mathrm{le} \mathrm{calcul} \mathrm{des} \mathrm{valeurs}$ intégrées de la production primaire ont été réalisés sur la couche $0-100 \mathrm{~m}$.

La production primaire a été mesurée après incubation in situ ct incorporation de traceur radioactif ${ }^{14} \mathrm{C}[25,60]$, à l'aide d'un appareillage semi-automatisé Let-Go [16, 17]. Cet appareil permet le prélèvement de l'échantillon et l'inoculation du radioélément simultanément in situ [12]. Les chambres à incubation ( $\sim 250 \mathrm{~mL})$ sont en polymétacrylate translucide pour les incubations claires (dix niveaux entre 5 et $100 \mathrm{~m}$ ) ou opaque pour les incubations à l'obscurité. Malgré la variabilité et l'importance de la fixation du carbone à l'obscurité [4], la mesure de celle-ci tous les deux niveaux est suffisante [9].

L'inoculation a été réalisée à l'aide d'une solution de bicarbonate de sodium marqué $\left(\mathrm{NaH}^{14} \mathrm{CO}_{3}\right.$; activité spécifique finale de $\left.17 \mu \mathrm{Ci} \mathrm{mL} \mathrm{mL}^{-1}\right)$. Les mesures ont été effectuées après des incubations courtes de 4 h (de 8 à 12 h GMT), entre février 1993 et juin 1994 (tableau $I$ ). Ces mesures constituent une bonne approximation de la production primaire brute (PPB) $[19,67]$, bien que de récents travaux de modélisation montrent que des problèmes de respiration préférentielle du carbone néoformé et/ou de recyclage intracellulaire pourraient modifier cette affirmation [68]. Les mesures s'échelonneraient alors entre la PPB et la production primaire nette en fonction du temps d'incubation et des conditions environnementales.

En fin d'incubation, le Let-Go est relevé manuellement. La quantité de radioactivité introduite est contrôlée dans chaque échantillon en prélevant $250 \mu \mathrm{L}$ d'eau de mer dans un flacon de $25 \mathrm{~mL}$ en polyéthylène contenant $250 \mu \mathrm{L}$ d'éthanolamine, $1 \mathrm{~mL}$ d'eau milliQ et $10 \mathrm{ml}$ de liquide scintillant aquasol (Dupont de Nemours). Les échantillons sont ensuite rapidement filtrés sous faible dépression ( $<5 \mathrm{~mm} \mathrm{Hg}$ ) sur des filtres GF/F $(25 \mathrm{~mm}$ de diamètre). Les filtres, préalablement recouverts par $250 \mu \mathrm{l}$ d' $\mathrm{HCl} 0,5 \mathrm{~N}$, sont séchés à l'étuve $\left(12 \mathrm{~h}\right.$ à $\left.40^{\circ} \mathrm{C}\right)$ puis placés dans des flacons $(25 \mathrm{~mL}$ en polyéthylène) contenant $10 \mathrm{~mL}$ de liquide scintillant aquasol.

L'utilisation des filtres GF/F est au centre de nombreuses polémiques concernant une éventuelle sous-estimation des mesures $[2,4,7]$. De récents travaux $[51,53,57]$ indiquent que l'utilisation des filtres GF/F entraînerait une sous-estimation de la biomasse particulaire de 10 à $50 \%$ selon le niveau trophique du milieu par rapport aux résultats obtenus à l'aide de filtres anodisc-anopore de porosité $0,2 \mu \mathrm{m}$. En terme de productivité $[2,57]$, cette sous-estimation serait inférieure à $10 \%$ dans les zones oligotrophes du Pacifique, voire négligeable en Méditerranée nord-occidentale [9].

Les comptages de radioactivité sont effectués au laboratoire, à l'aide d'un compteur à scintillation liquide de 


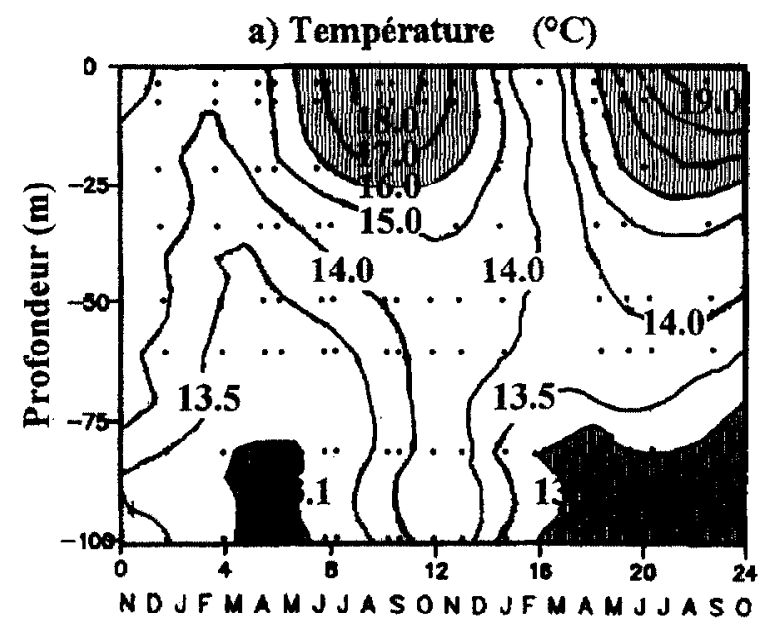

1993

1994

b) nitrate $(\mu \mathrm{M})$

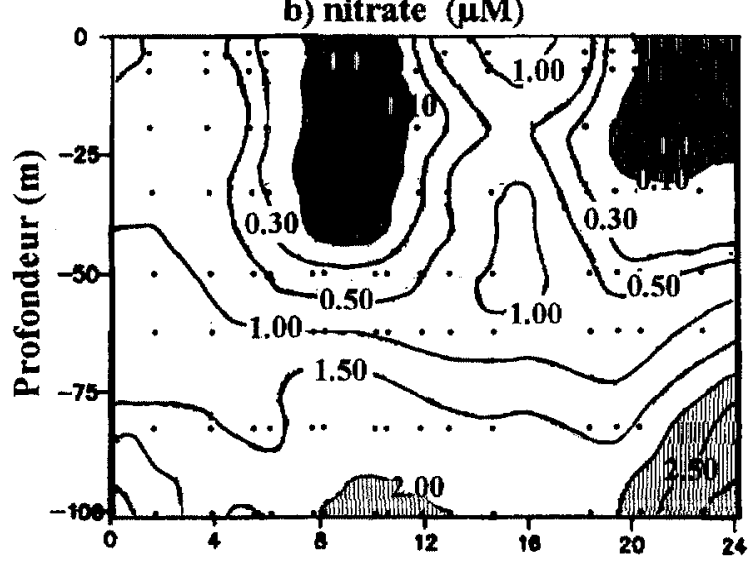

c) Chlorophylle (mg.m ${ }^{-3}$ )

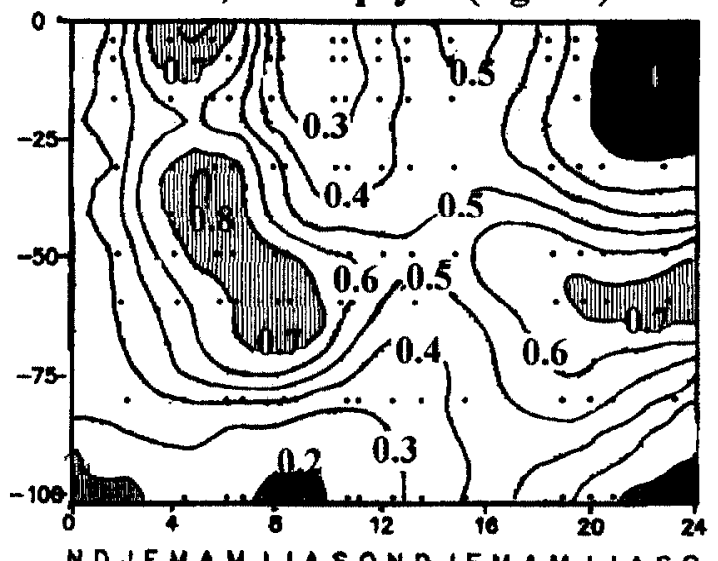

1993

1994

d) Production primaire $\left(\mathrm{mg} \cdot \mathrm{m}^{-3} \cdot \mathrm{h}^{-1}\right.$ de $\left.\mathrm{C}\right)$



Figure 1. Évolution temporelle entre novembre 1992 et novembre 1994, à la station M3, de a) température potentielle $\left({ }^{\circ} \mathrm{C}\right)$, b) nitrate $(\mu \mathrm{M})$, c) chlorophylle $\left(\mathrm{mg} \mathrm{m}^{-3}\right)$, d) production primaire $\left(\mathrm{mg} \mathrm{m} \mathrm{m}^{-3} \mathrm{~h}^{-1}\right.$ de $\mathrm{C}$ ). Les mlaxima (clair) et les minima (sombre) sont identifiés sur les coupes.

Figure 1. Time series between November 1992 and November 1994 , at station $\mathrm{M} 3$ of a) potential temperature $\left({ }^{\circ} \mathrm{C}\right)$, b) nitrate $(\mu \mathrm{M})$, c) chlorophyll $\left(\mathrm{mg} \mathrm{m}^{-3}\right)$, d) primary production $\left(\mathrm{mg} \mathrm{m}^{-3} \mathrm{~h}^{-1}\right.$ of $\mathrm{C}$ ). Maxima (light) and minima (dark) are identified on the transects.

type Beckman. La quantité de carbone fixé par unité de temps est calculéc selon la formule :

$P P B=\frac{(d p m e-d p m b)}{d p m i} \times \frac{V p}{V f} \times A \times 1,05 \times \frac{1}{t}$

avec : PPB $=$ production primaire brute $\left(\mathrm{mg} \mathrm{m}^{-3} \mathrm{~h}^{-1}\right.$ de C) , dpme $=$ dpm (désintégrations par minute) mesurés dans l'échantillon ; dpmb $=$ dpm mesurés dans l'échantillon incubé à l'obscurité ; dpni = dpm introduit dans l'échantillon ; $\mathrm{Vp}=$ volume prélevé dans l'échantillon $(0,25 \mathrm{~mL}) ; \mathrm{Vf}=$ volume filtré $(\mathrm{en} \mathrm{mL}) ; \mathrm{A}=$ concentra- tion des carbonates dans l'eau de mer $\left(\sim 25000 \mathrm{mg} \mathrm{m}^{-3}\right)$; $1,05=$ fractionnement isolopique entre ${ }^{12} \mathrm{C}$ et ${ }^{14} \mathrm{C} ; \mathrm{t}=$ temps d'incubation (en heures).

\section{RÉSULTATS}

\subsection{Approche méthodologique}

L'estimation d'un bilan réaliste de la productivité de la zone nécessite l'introduction d'un facteur correctif $\mathrm{Fc}$, afin de transformer les estimations horaires en produc- 
Tableau I. Dates de mesures de la production primaire et programmes concernés $(\mathrm{SVL}=$ Suivilion, $\mathrm{PRO}=$ Procepel).

Table I. Dates of primary production measurements and evolved programs (SVL= Suivilion, $\mathrm{PRO}=$ Procepel).

\begin{tabular}{rlrl}
\hline \multicolumn{1}{l}{ Date } & Prog. & \multicolumn{1}{c}{ Date } & Prog. \\
\hline 25 févr. 1993 & PRO01 & 6 nov. 1993 & PRO05 \\
15 avr. 1993 & SVL02 & 6 déc. 1993 & SVL06 \\
3 mai 1993 & PRO02 & 22 janv. 1994 & PRO07 \\
19 juin 1993 & SVL03 & 17 févr. 1994 & SVL07 \\
2 juil. 1993 & PRO03 & 2 mai 1994 & SVL08 \\
1 sept. 1993 & SVL04 & 1 juin 1994 & PRO09 \\
17 sept. 1993 & PRO04 & 27 juin 1994 & SVL09 \\
20 oct. 1993 & SVL05 & & \\
\hline
\end{tabular}

tions journalières [9]. En effet, Rodhe [55] signale des variations de 20 à $30 \%$ dans les fixations de ${ }^{14} \mathrm{C}$ entre des incubations de $4 \mathrm{~h}$ ramenées à $12 \mathrm{~h}$ et des incubations effectives de $12 \mathrm{~h}$. Ce facteur présente des variations spatio-temporelles importantes. Dans le Pacifique nord, une variation de 12 à $15 \%$ [23] ou de $25 \%$ [46] est observée entre des incubations de $6 \mathrm{~h}$ ramenées à $24 \mathrm{~h}$ et des incubations de $24 \mathrm{~h}$. Le facteur correctif proposé par les auteurs varie de 0,66 à 1,30 . Il est inférieur à 1,00 entre mars et juin et est maximal en février. Un facteur correctif $\mathrm{Fc}$ moyen a été estimé à partir d'expériences réalisées en juin-juillet, à la station M3 et à la station S5 au large des îles Baléares [9]. Deux systèmes Let-Go ont été déployés simultanément, puis relevés respectivement après $4 \mathrm{~h}$ et $12 \mathrm{~h}$ d'incubation. Les profils obtenus permettent de visualiser les variations verticales de $\mathrm{Fc}$ (figure 2). Minimal en surface $(\sim 0,6$ à 0.8$)$, le facteur est proche de 1,0 vers $60 \mathrm{~m}$ et au-delà, sous la couche euphotique. Cette distribution est cohérente avec la variations des effets journaliers de la lumière, maximaux en surface et diminuant avec la profondeur [47].

En terme de quantité intégrée sur la couche 0-100 m, les bilans de la production primaire varient d'un facteur de 0,7 à 0,8 entre les incubations de $4 \mathrm{~h}$ (multipliées par trois) et de $12 \mathrm{~h}$. Une valeur moyenne de 0,75 est adoptée pour celte étude (en accord avec les échelles proposées par [55 et 46]). La correction est appliquée aux quantités intégrées de carbone sur la couche $0-100 \mathrm{~m}$ pour chaque sortie, de manière à minimiser l'erreur liée aux variations verticales du facteur. Soit $T$, la durée en heure de l'éclairement journalier, alors :

PPB $\left\{\mathrm{mg} \mathrm{m}^{-2} \mathrm{~d}^{-1} \mathrm{de} \mathrm{C}\right\}=\mathrm{PPB}_{\text {incu }}\left\{\mathrm{mg} \mathrm{m}^{-2}[4 \mathrm{~h}]^{-1} \mathrm{de} \mathrm{C}\right\}$ * $3 * \mathrm{Fc} *(\mathrm{~T} / 12)$

\subsection{Variations spatio-temporelles}

\subsubsection{La structure thermique (figure 1a)}

- Cycle saisonnier : l'évolution de la températurc dans la couche de surface $0-100 \mathrm{~m}$ est classique pour la région $[35,40,41,42]$. Chaque année est marquéc par une alternance entre l'homogénéisation thermique en hiver et la stratification en été [43]. La phase d'homogénéisation débute au cours des mois d'octobre-novembre et se termine en mars avril, occasionnellement en mai. Elle se traduit par des minima de température dans la couche $0-100 \mathrm{~m}\left(<13,1^{\circ} \mathrm{C}\right)$ et par la disparition des isothermes supérieures ou égales à $15,0^{\circ} \mathrm{C}$ en surface (figure $\mathrm{I} a$ ).

Dès les mois de mars-avril, le réchauffement superficiel de la colonne d'eau entraîne l'apparition d'une stratification thermique. Celle-ci se renforce au cours de l'été, particulièrement dans les 25 premiers mètres où les températures dépassent $18,0^{\circ} \mathrm{C}$ (figure la). Une thermocline saisonnière apparaît à la fin du mois d'avril, se renforce et s'enfonce, puis disparaît en octobre sous l'effet des mélanges turbulents.

- Variabilité : d'importantes variations interannuelles sont rencontrées au cours des phases d'homogénéisation, en rapport avec les conditions atmosphériques plus ou moins rudes de la saison. Ainsi, en janvier-février 1993, l'isotherme $14,0^{\circ} \mathrm{C}$ n' atteint pas la surface au cours des différentes prospections, alors qu'à la même période en 1994, les températures mesurées en surface sont inférieures à $13,5^{\circ} \mathrm{C}$ à la station M3 (figure la). Les vents plus violents et principalement de secteur nord-nordouest fournissent une explication satisfaisante à ces varialions $[9,13]$.

\subsection{2. Évolution des sels nutritifs (figure $1 \mathrm{~b}$ )}

- Cycle saisonnier : au cours de l'hiver, l'homogénéisation de la colonne d'eau par convections thermohalines induit l'apport de quantités importantes d'éléments nutritifs dans la couche superficielle [15]. Ainsi, l'isoplèthe $1,0 \mu \mathrm{M}$ en $\mathrm{NO}_{3}$ atteint la surface en décembre. Les concentrations en phosphate (non présentées ici) suivent la même évolution saisonnière que les concentrations en nitrate.

Dès le mois de mars, les concentrations en sels nutritifs (e.g. figure $I b$ pour le nitrate) diminuent à la suite de la consommation biologique. En surface, cette consommation est rapide et, dès le mois de juin, les concentrations en nitrale sont faibles $(<0,10 \mu \mathrm{M})$ puis indétectables par 



Figure 2. Distribution verticale du facteur de correction Fc, entre des incubations réalisées sur $4 \mathrm{~h}$ et sur $12 \mathrm{~h}$. Incubations réalisées à la station M3 (échelle en bas) et au large des Baléares ( $\mathrm{S} 5$, échelle d'en haut) à l'aide de 2 Let-Go, l'un relevé au bout de $4 \mathrm{~h}$ (---), l'autre après $12 \mathrm{~h}$ (--). Les histogrammes (à gauche) montrent la valeur du facteur Fc pour chaque profondeur.

Figure 2. Vertical distribution of the factor Fc, for (---) short (4 h) to (-) long ( $12 \mathrm{~h}$ ) incubatjons carried out at station M3 (botlon scale) and near Balearic Islands (S5, top scale) with 2 Let-Go systems. Histograms (on the left) characterise the value of the Fc factor for each depth.

les méthodes de mesure classiques. La consommation biologique du nitrate, couplée à une remontée d'eau plus riche en nutriments, identifiée par l'isoplèthe $2,0 \mu \mathrm{M}$ de nitrate à $100 \mathrm{~m}$ (figure $1 \mathrm{~b}$ ), provoque l'apparition d'une nitracline puis intensifie le gradient. Le sommet de la nitracline (figure 3) atteint une profondeur moyenne de 40-60 $\mathrm{m}$ avant de disparaître au début de l'hiver (figures $1 b, 3$ ).
- Variabilité : ce schéma général de la répartition verticale du nitrate au cours de l'année présente d'importantes variations interannuelles et spatiales. Ainsi, les mélanges plus intenses au cours de l'hiver 1993-1994 (H93) se traduisent par des enrichissements plus importants en nitrate dans la couche superficielle par rapport à H92 et sont suivis en été (E94) par une intensification des gradients en nitrate par rapport à E93 (figure 1b). Cette intensification 


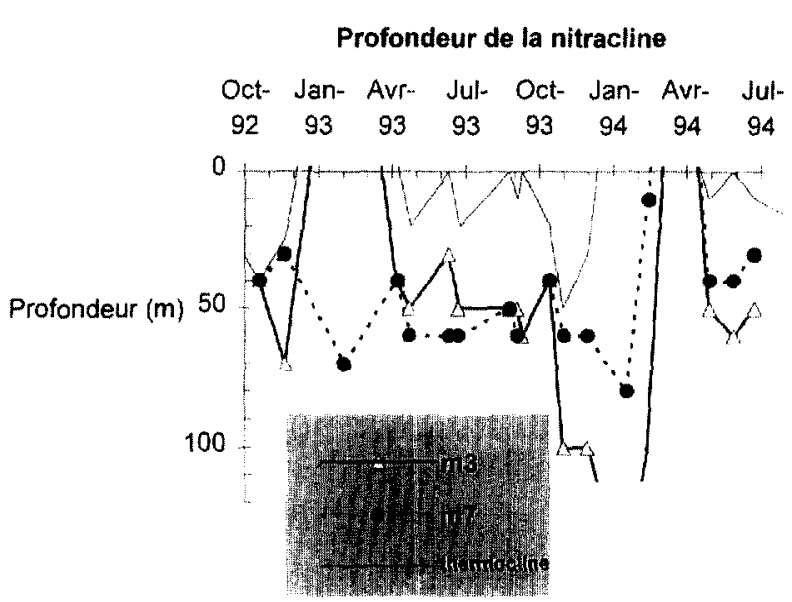

Figure 3. Évolution temporelle de la profondeur de la nitracline aux stations M3 (- $-\mathbf{-}$ ) et M7 (-- --) et de la profondeur de la thermocline (-) à la station M3

Figure 3. Time series of the depth of the nitracline at stations M3 $(-\triangle-)$ and M7 (-- -$)^{-}$) and of the thermocline (- - ) at station M3.

est indiquée par la présence de l'isoplèthe $3,0 \mu \mathrm{M}$ en nitrate à $100 \mathrm{~m}$ en septembre 1994 , au lieu de l'isoplèthe 2,0 $\mu \mathrm{M}$ à la même profondeur en 1993. Au cours de cet été (E94), la couche épuisée en nitrate est moins épaisse $(25-30 \mathrm{~m})$ qu'en $\mathrm{E} 93(40 \mathrm{~m})$.

Parallèlement à la variabilité temporelle, il est possible d'observer une variabilité spatiale des mélanges turbulents et de la répartition des éléments nutritifs sur la colonne d'eau. Lors des mélanges verticaux moins intenses de l'hiver 1992, la nitracline persiste à la station M7, contrairement à la station M3 plus côtière (figure 3). Par contre, pendant l'hiver 1993, les mélanges turbulents entraînent la disparition de la nitracline à la station M3 et à la station M7.

\subsection{3. Évolution de la biomasse chlorophyllienne et de la production primaire (figures $1 \mathrm{c}$ et $1 \mathrm{~d}$ )}

- Cycle saisonnier : les concentrations hivernales en chlorophylle (figure $1 c$ ) et les valeurs de la production primaire (figure $1 d$ ) sont homogènes et fortes dans la couche $0-80 \mathrm{~m}$, avec respectivement $0,4 \mathrm{mg} \mathrm{m}^{-3}$ et entre 0,3 et $0,8 \mathrm{mg} \mathrm{m}^{-3} \mathrm{~h}^{-1}$ de $C$.

Dès les mois de mars-avril, les concentrations en chlorophylle augmentent (isolignes 0,7 et $0,8 \mathrm{mg} \cdot \mathrm{m}^{-3}$ et maxima supérieurs à $2,0 \mathrm{mlg} \mathrm{m}^{-3}$ ) et caractérisent l'efflorescence printanière [30], principalement dans la couche de surface (figure $/ \mathrm{c}$ ). Parallèlement, la production pri- maire augmente $\left(>1,0 \mathrm{mg} \mathrm{m}^{-3} \mathrm{~h}^{-1}\right.$ de $\left.\mathrm{C}\right)$ dans cette même couche superficielle (figure $1 d$ ). Malgré un mauvais échantillonnage de l'efflorescence printanière en 1994 en raison de mauvaises conditions météorologiques, l'auginentation de la production primaire est bien marquée jusqu'en juin (isoligne $1,6 \mathrm{mg} \mathrm{m}^{-3} \mathrm{~h}^{-1} \mathrm{de}$ C).

La situation estivale se caractérise par une diminution drastique des concentrations en chlorophylle $(<0,3$ voire $<0,1 \mathrm{mg} \mathrm{m}^{-3}$ ) dans les eaux superficielles (figure $1 \mathrm{c}$ ), mais par une relative constance et homogénéité $(\sim 0,8$ à $1,0 \mathrm{mg} \mathrm{m}^{-3} \mathrm{~h}^{-1}$ de C) des valeurs de la production primaire (figure $I d$ ). Les concentrations maximales en chlorophylle $\left(0,5\right.$ à $\left.0,7 \mathrm{mg} \mathrm{m}^{-3}\right)$ sont rencontrées plus profondément $(60-70 \mathrm{~m})$. Elles caractérisent le Maximum Profond de Chlorophylle (MPC), mais sont accompagnées d'une légère diminution de la production primaire. Au-delà de $80 \mathrm{~m}$, les concentrations en chlorophylle $\left(<0,3 \mathrm{mg} \mathrm{m}^{-3}\right)$ et les valeurs de la production primaire $\left(<0,2 \mathrm{mg} \mathrm{m}^{-3} \mathrm{~h}^{-1}\right.$ de $C$ ) restent faibles tout au long de la saison estivale (figures $l c$ et $l d$ ).

De manière à étendre les précédentes descriptions du cycle saisonnier de la chlorophylle et à préciser la variabilité spatiale de la biomasse autotrophe, il est utile de replacer les observations effectuées à la station fixe M3 dans la structure plus synoptique de la radiale (figure $4 a$ ). Malgré l'influence des mouvements ondulatoires du CNM [13] sur le système autotrophe [9], des répartitions types en été et en hiver de la chlorophylle peuvent être proposées (figure 4b). La première (septembre 1994) est caractérisée par des maxima profonds (MPC) au large et près des côtes. La seconde (février 1994) est marquée par une homogénéité verticale et présente des concentrations maximales sur ou à proximité du plateau continental.

- Variabilité : la variabilité interannuelle est forte en termes de chlorophylle et de productivité du système. Les valeurs des deux variables dans la couche de surface (figures $1 c, 1 d$ ) sont plus fortes en hiver 1993 qu'en hiver 1992, malgré des conditions de turbulcnces plus intenses [9] détruisant les gradients par une homogénéisation plus poussée de la colonne d'eau (figure 3).

Les variations estivales sont marquées, entre l'été 1993 et l'été 1994, par des concentrations en chlorophylle plus faibles dans la couche de surface et par une relative constance du MPC en concentration et en profondeur au cours de l'été 1994 (figure /c), malgré les fortes variations en profondeur et en intensité des gradients en sels nutritifs. À l'opposé, les valeurs de la production primaire sont nettement plus fortes en surface au début de l'été 1994, 


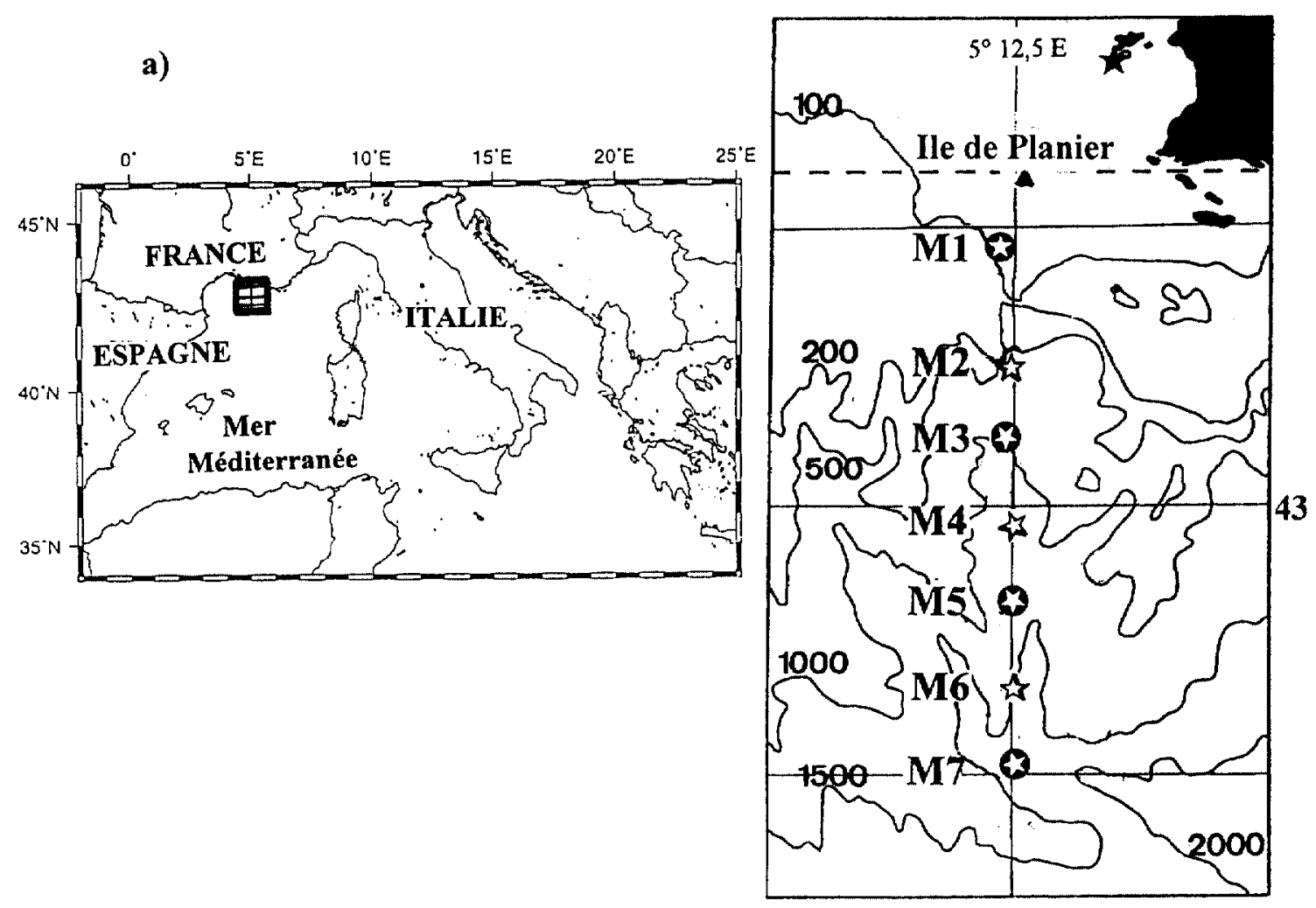

b)

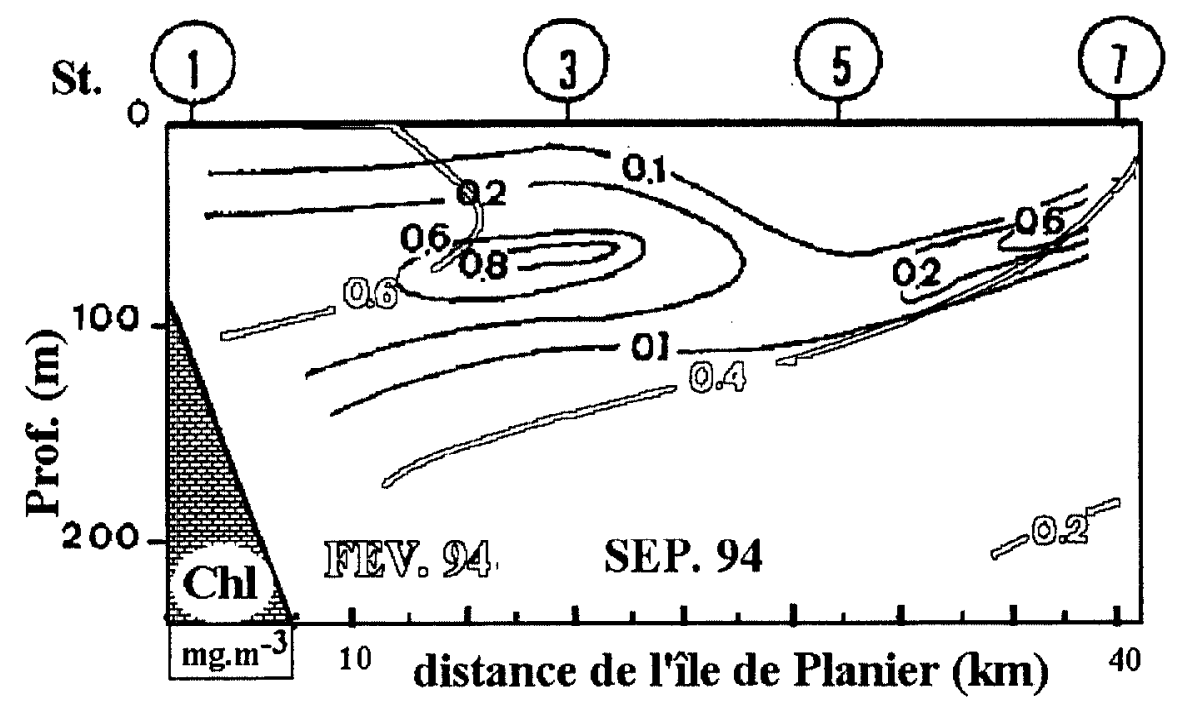

Figure 4. a) Carte extraite de Conan et al. [13], Fig. 2. b) Superposition de la distribution de la chlorophylle (mg $\mathrm{m}^{-3}$ ), au cours d'une situation hivernale (février) et en fin de saison estivale (septembre).

Figure 4. a) Map extracted from Conan et al. [13], Fig. 2. b) Superimposes distributions of chlorophyll (mg $\mathrm{m}^{-3}$ ) for winter (February) and late summer (September). 
en relation avec la plus forte abondance des sels nutritifs (figures $1 b, I d$ ).

\section{DISCUSSION}

L'étude des relations entre les phénomènes biologiques et les structures dynamiques à mésoéchelle dans le golfe du Lion contribue à une meilleure compréhension du fonctionnement des écosystèmes littoraux. La collecte de données expérimentales est une étape essentielle pour une modélisation prédictive fiable des écosystèmes et pour la réduction des incertitudes quant au rôle des régions côtières en tant que source [58] ou puits [56] de carbone. Il s'agit de quantifier la productivité des régions côtières et de caractériser les flux de matière dans l'écosystème et aux interfaces (objectifs du Programme national d'océanographie côtière).

a)



b)

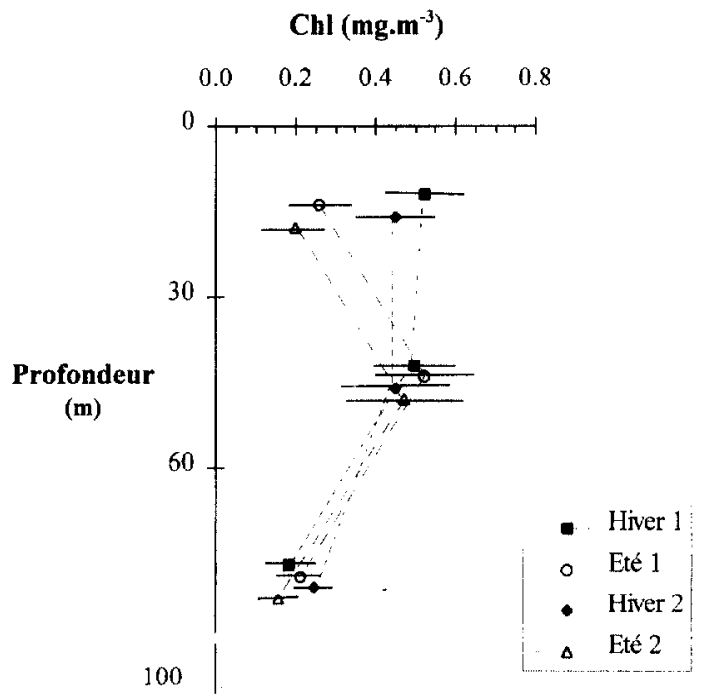

\subsection{Caractérisation spatiale de la zone d'étude}

L'analyse de l'hydrologie (figure $1 a[9,13]$ ) a permis de définir deux saisons par opposition aux quatre saisons climaliques généralement retenues : une saison hydrologique hivernale de novembre à début mai, marquée par une colonne d'eau homogène et turbulente, puis une saison hydrologique estivale de mi-mai à fin octobre, marquée par une stratification de plus en plus poussée de la colonne d'eau.

La radiale prospectée est divisée en trois zones distinctes en considérant les concentrations annuelles moyennes en chlorophylle des stations (figure $5 a$ ) : (1) une zone côtière riche $\left(>0.55 \mathrm{mg} \mathrm{m}^{-3}\right)$ à la station M1; (2) une zone plus pauvre $\left(<0,35 \mathrm{mg} \mathrm{m}^{-3}\right)$ à la station M5, sous l'influence des eaux oligotrophes du CNM : (3) deux zones intermédiaires $\left(0,43\right.$ et $\left.0,40 \mathrm{mg} \mathrm{m}^{-3}\right)$ aux stations $\mathrm{M} 3$ et M7, subissant l'influence alternative des eaux du CNM et

Figure 5. a) Moyennes et intervalles de confiance (95\% basé sur l'écart type groupé) des concentrations en chlorophylle pour chaque station de la radiale au cours du suivi. Les moyennes à la station $M 1$ et M5 sont significativement différentes $(p<0,01$ ) de la moyenne générale. b) Comme a) après transformation logarithmique pour stabiliser la variance des observations, en fonction de la saison et par couche $(0-30 \mathrm{~m}, 30-60 \mathrm{~m}$ et $60-100 \mathrm{~m}$; les moyennes sont représentées décalées sur la verticale). Les différences des moyennes sont significatives $(p<0,01)$ dans la couche de surface entre l'hiver et l'été et entre couches successives en été.

Figure 5. a) Mean and $95 \%$ confident level (based on pooled standard deviation) chlorophyll concentrations for each station of the transect during the sampling period. Mean values of stations M1 and M5 are significantly different from the total mean value $(p<0.01)$. b) As in a) after a logarithmic transformation, by seasons and by layers $(0-30 \mathrm{~m}, 30-60 \mathrm{~m}$ and $60-100 \mathrm{~m}$; Values are given with a weak depth gap). The differences are significant $(p<0.01)$ in the surface layer between winter and summer and between successive layers in summer. 
d'eau plus riche, issue soit de la divergence du large pour $\mathrm{M} 7[42,45]$, soit de la région côtière pour M3 $[8,11,52]$.

Afin de synthétiser les résultats concernant la répartition verticale et saisonnière de la chlorophylle à la station M3 et d'interpréter sa dynamique, trois couches successives $0-30 \mathrm{~m}, 30-60 \mathrm{~m}$ et $60-100 \mathrm{~m}$ ont été définies (figure $5 b$ ). La variation des moyennes saisonnières sur chaque couche caractérise le cycle décrit précédemment. mais montre également que la formation du MPC [27] est à rapprocher d'une diminution des concentrations dans la couche de surface $(0-30 \mathrm{~m})$, plutôt qu'à une augmentation significative des concentrations en profondeur ( 30 $60 \mathrm{~m}$ ). Ces résultats révèlent également une limite du modèle proposé par Lévy et al. [38] qui prédit une biomasse phytoplanctonique minimale au cours de la saison hivernale en relation avec les turbulences et l'épaisseur importante de la couche de mélange, alors que nos mesures indiquent des maxima en chlorophylle importants en hiver.

\subsection{Facteurs contrôlant le système productif}

L'échantillonnage mensuel permet de saisir la variabilité à mésoéchelle de la productivité de la zone (figure 6) en relation avec l'hydrodynamisme [61]. Zakardjian [69] considère que deux régimes hydrologiques, l'un à faibles et l'autre à fortes turbulences et flux diffusifs, suffisent

A)

1

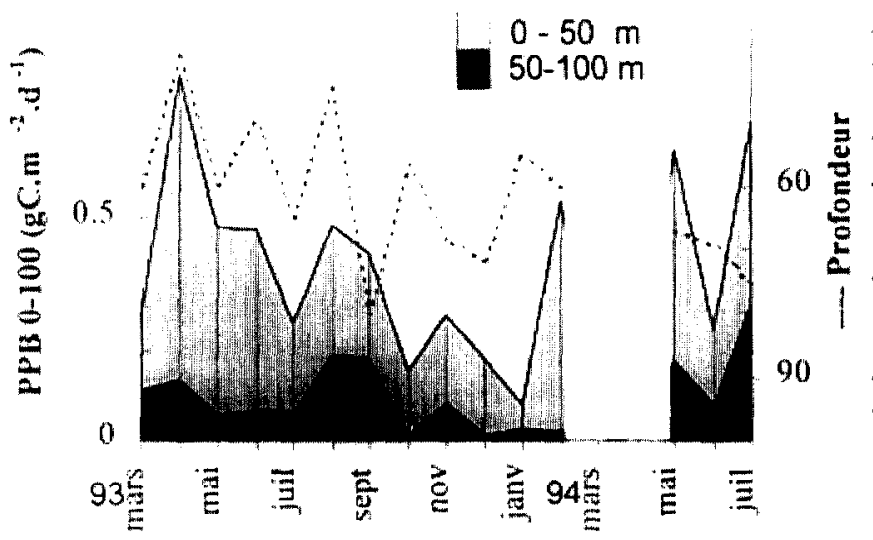

30

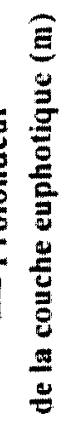

B)
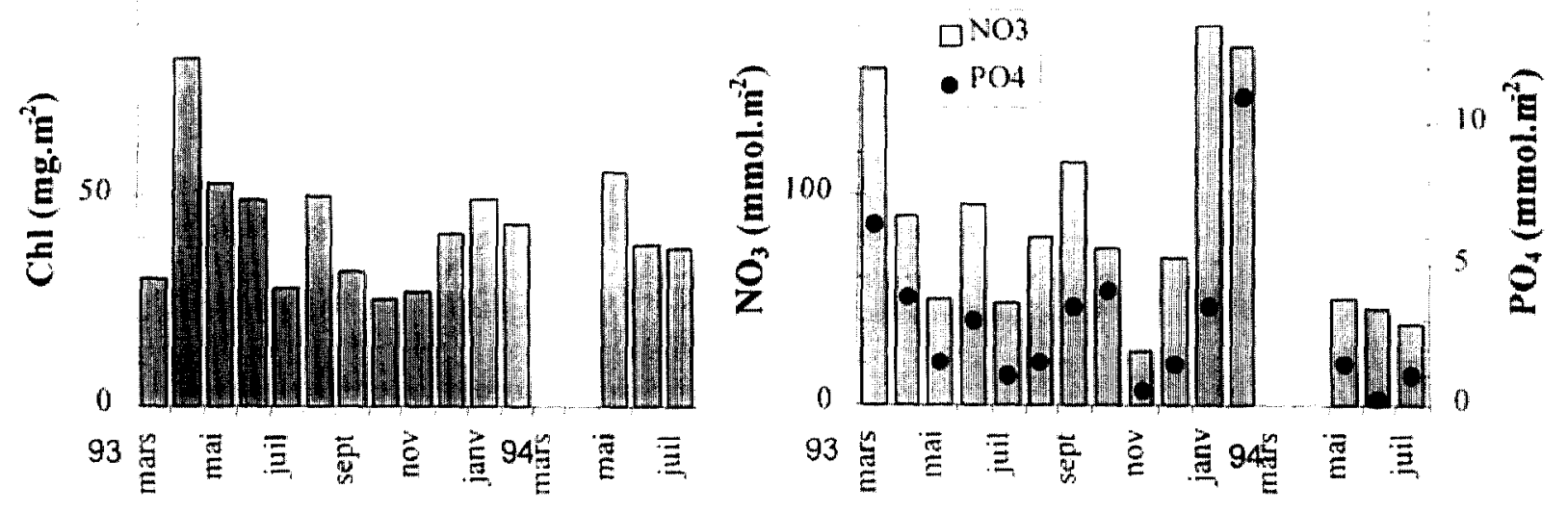

Figure 6. Évolution temporelle $\mathbf{A}$ ) de la profondeur de la couche euphotique et des quantités intégrées de la production primaire $\left(\mathrm{mg} \mathrm{m}^{-2} \mathrm{~d}^{-1}\right.$ de C), B) des quantités intégrées $(0-100 \mathrm{~m})$ de la chlorophylle $\left(\mathrm{mg} \mathrm{m}^{-2}\right)$, du nitrate et du phosphate $\left(\mathrm{mmol} \mathrm{m}^{-2}\right)$.

Figure 6. Time series of $\mathbf{A})$ depth of the euphotic zone and integrated primary production $\left(\mathrm{mg} \mathrm{m}^{-2} \mathrm{~d}^{-1}\right.$ of $\left.\mathrm{C}\right)$, B) integrated $(0-100 \mathrm{~m})$ chlorophyll $\left(\mathrm{mg} \mathrm{m}^{-2}\right)$, nitrate and phosphate $\left(\mathrm{mmol} \mathrm{m}^{-2}\right)$. 
pour expliquer la distribulion des nutriments et de la biomasse planctonique en Méditerranée nord-occidentale, tandis que Lévy el al. [38] considèrent six situations différentes pour modéliser la productivité de la zone et les quantités de matière exportée.

La description des cycles saisonniers des sels nutritifs, de la chlorophylle et de la production primaire justifie une division en trois situations distinctes, les deux premières intervenant au cours de l'hiver hydrologique, la dernière au cours de l'été hydrologique (tableau $I$ ). Ces situations sont respectivement (1) une situation hivernale, (2) une situation hivernale tardive d'efflorescence (correspondant au bloom printanier), (3) une situation estivale. Dans chacun de ces systèmes, la production primaire est sous la dépendance d'un ou de la combinaison de plusieurs facteurs $[3,26,33]$ tels que la biomasse chlorophyllienne [27, 54], les sels nutritifs $[11,37,42]$, l'éclairement [47], la température [63] ou la turbulence $[15,34,64]$.

(1) Au cours de l'hiver, les conditions thermiques et lumineuses ne sont pas réellement limitantes pour les communautés phytoplanctoniques en Méditerranée occidentale [15, 59]. Le contrôle par la turbulence est plus difficile à mettre en évidence compte tenu de sa variabilité spatiale et temporelle. Par exemple, au cours de l'hiver 1992, les mélanges restent localisés au large et la couche de mélange à la station M3 reste peu épaisse (figure 3). Une association entre les vitesses du CNM comprises entre 5 et $20 \mathrm{~cm} \mathrm{~s}^{-1}$ et les maxima de chlorophylle a été mise en évidence le long de la radiale $[9]$.

Un flux advectif plus ou moins permanent $[13,59]$ et la présence d'une zone frontale à la station M3 peut expli. quer les concentrations plutôt élevées en chlorophylle malgré l'épaisseur parfois importante de la couche de mélange $(200-400 \mathrm{~m})$. La production primaire reste forte au cours de l'hiver (figures $1 d, 6 a$; tableau $I$ ) et témoigne d'une bonne capacité d'adaptation du phytoplancton aux turbulences $[54,64]$, surtout que le zooplancton maintient une forte activité de prédation au cours de cette saison [44].

Les mesures réalisées au cours de la période hivernale doivent pourtant être considerées avec précaution. En effet, l'échantillonnage est discret dans l'espace et dans le temps et les sorties sont effectuées lors d'une accalmie relative des conditions météorologiques, plusieurs heures après l'arrêt du forçage atmosphérique [10]. Ce délai pourrait permettre le développement d'efflorescences algales, dont l'impact serait réduit dans l'espace et le temps, mais important en tcrme de flux de matière dans les zones considérées. Nos estimations de biomasse et de production intégrées seraient alors surestimées et expliqueraient les maxima de chlorophylle observés pendant l'hiver.

(2) Dans le golfe du Lion, l'efflorescence algale à la fin de l'hiver hydrologique précède l'établissement de la thermocline (figures $1 a, l c$, tableau $I I$ ) comme l'ont déjà observée Townsend et al. [63] en Atlantique. Cette observation est en accord avec les capacités d'adaptation rapide du phytoplancton. Ce développement de la chlorophylle en surface entraîne une diminution rapide des réserves nutritives, clairement retrouvée sur les valeurs discrètes (figures $/ b$ ) et intégrées (figure 6b). C'est également au cours de cette période que les maxima en production primaire [31] sont mesurés (figures $1 d, 6 a$ ). Ces observations sont particulièrement importantes en terme de bilan (tableau $I I$ ) puisque, parallèlement au maximum de productivité, les flux du CNM sont maximaux [13] et la colonne d'eau peu stratifiée. Ceci se traduit par d'importants flux d'exportation de matière par advection horizontale et verticale [21].

Le rôle de la lumière s'avère complexe au cours de cette période. Malgré des concentrations fortes en chlorophylle sur une couche de plus de $60 \mathrm{~m}$, les valeurs de la production primaire n'augmentent que dans les 30 ou 40 premiers mètres (figures $1 c, l d$ ). L'influence des concentrations en pigments sur la colonne d'eau pourrait modifier la quantité et la qualité de la lumière disponible pour le phytoplancton localisé plus en profondeur, selon un phénomène d'auto-ombrage $[27,47]$.

(3) La situation estivale est caractérisée par un MPC bien défini (figure $5 b$, tableau $I I$ ). La formation de ce dernier peut être reliée à un phénomène d'adaptation du phytoplancton aux conditions lumineuses par augmentation des teneurs en chlorophylle mais sans accroissement proportionnel de l'efficacité photosynthétique $[4,28,29,54]$. La lumière est alors un facteur important, limitant la production du MPC. En effet, des phénomènes de diffusion turbulente à travers la nitracline $[27,28]$, de pulses sous l'effet du déplacement d'ondes internes [34], ainsi que les phénomènes de nitrification [18] assureraient un apport constant de nitrate, à l'origine d'une production nouvelle importante dans le MPC par rapport au reste de la colonne d'eau [24].

À l'opposé, darss la couche de surface, les concentrations en chlorophylle diminuent et les réserves nutritives sont épuisées (figures $l b, l c, 6 b ;$ tableau $I I$ ). La limitation de 
Tableau II. Représentation schématique et description des profils de la production primaire ( $\mathrm{PPB}$ ), chlorophylle ( $\mathrm{Chl}$ ), nitrate ( $\left.\mathrm{NO}_{3}\right)$, température $(T)$ et lumière $(L)$ en fonction de trois saisons définies dans le texte $=(1),(2)$, (3). Homo: distribution homogène, Augm. : augmentation des valeurs, Cons. : consommation biologique, Min. : minimum, Max. : maximum, Z : profondeur, clas : décroissance exponentielle classique de la lumière, ce : profondeur $(m)$ de la couche euphotique, : pas de changement important.

En bas : Valeur par saison et contribution par couche au bilan annuel (tot.) de la production primaire brute.

Table II. Schematic representation and description of the vertical profils of primary production ( $\mathrm{PPB})$, chlorophyll $(\mathrm{Chl})$, nitrate $(\mathrm{NO})$, temperature ( $\mathrm{T}$ ) and light ( $\mathrm{L}$ ) according to the three seasons defined in the text $=(1),(2),(3)$. Homo: homogeneous distribution, Augm.: increase of the values, Cons.: biological uptake, Min.: minimum, Max.: maximum, Z: depth, clas: classical light exponential decrease, ce: depth (m) of the euphotic layer, : no significant change.

Bottom: Contribution by season and by layer to the annual budget of the total primary production.

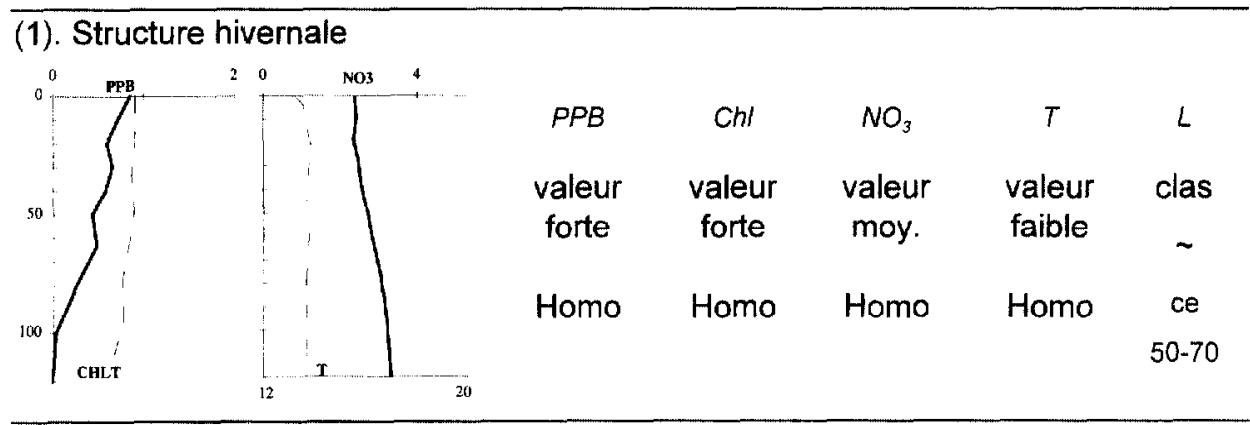

(2). Structure d'efflorescence à la fin de l'hiver



\section{(3). Structure estivale}
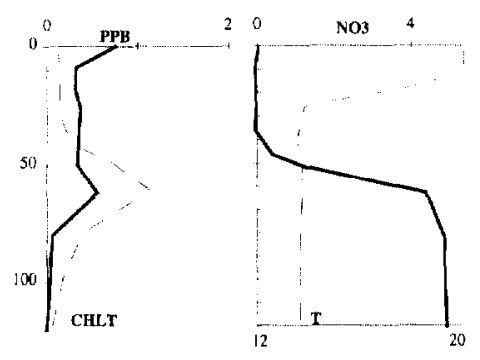



$\mathrm{NO}_{3}$
$\sim 0$ en
surface
forte
nitracline
(diffusion)

$\begin{array}{cc}T & L \\ \text { Max. } & \sim \\ \text { annuels } & \\ \text { forte } & \text { ce } \\ \text { thermocline } & 40-70 \\ \text { superficielle } & \end{array}$

\begin{tabular}{lcccc} 
couche/structure & $(1)$ & $(2)$ & $(3)$ & Annuel \\
$0-100 \mathrm{~m}$ PPB & \multicolumn{2}{c}{$80-85$} & $60-65$ & $140-150$ \\
$\left(\mathrm{~g} \cdot \mathrm{m}^{-2} \cdot \mathrm{a}^{-1} \mathrm{de}\right.$ C) & & & & \\
$0-50 \mathrm{~m}$ PPB & $60-90 \%$ & $>90 \%$ & $50-65 \%$ & $75 \%$ \\
$50-100 \mathrm{~m}$ PPB & $10-40 \%$ & $<10 \%$ & $35-50 \%$ & $25 \%$ \\
\hline
\end{tabular}


la production primaire par les sels nutritifs est significative $[38,44,65]$. Pourtant, les valeurs de production primaire restent relativement fortes (figures $I d, 6 a$ ) mais traduisent sans doute un changement de nature de la production primaire, devenant alors principalement supportée par des processus de régénération. Le rapport $\mathrm{PPB} /$ Chl caractérise l'efficacité de production du phytoplancton $[29,36,49]$. Ce rapport diminue rapidement au cours de la période estivale pour atteindre des valeurs inférieures à $0,5 \mathrm{mg}(\mathrm{mg} \mathrm{Chl})^{-1} \mathrm{~h}^{-1}$ de C. Cette diminution se traduit par une augmentation drastique du rapport entre la production bactérienne et la production primaire.

La zone de la station M3, près des accores du plateau continental, pourrait être plus productive que les zones plus au sud du golfe du Lion, ou même plus côtières [11, $31,33]$ sous l'effet de phénomènes hydrodynamiques assurant un enrichissement : dynamique frontale (bord du CNM), circulation secondaire, remontée d'eau le long de la pente continentale. apports terrigènes superficiels $[9,13]$.

\subsection{Bilan et contribution par couche ou par saison}

L'établissement du bilan de la productivité à la station M3 est effectué par l'intégration des différentes variables sur une colonne d'eau 0-100 m (figure 6), c'est-à-dire sur une épaisseur supérieure à la couche euphotique mais plus proche de l'épaisseur réelle de la couche productive [47]. Les précédentes estimations étaient généralement limitées aux 50 ou 60 premiers mètres $[37,42]$ ou déduites de mesures satellitales [48].

Le bilan annuel de la production primaire à l'entrée du golfe du Lion est compris entre $140 \mathrm{et} 150 \mathrm{~g} \mathrm{~m}^{-2} \mathrm{a}^{-1}$ de C. Cette valeur est sensiblement plus élevée que celles citées dans la littérature $[37,42]$ mais s'explique par les raisons évoquées ci-dessus et/ou par des différences d'ordre méthodologique :

- L'échantillonnage mensuel adopté dans cette étude est plus apte à cerner la variabilité importante de la production. Ies précédents bilans annuels ne s'appuyaient que sur quatre ou six mesures.

- L'ulilisation du système Let-Go permet de s'affranchir de nombreux problèmes de pollution et de perturbation de l'échantillon [50]. Ce système foumil des valeurs supérieures de 10 à $20 \%$ par rapport aux méthodes d'incubation in situ classiques en Atlantique [16] ou ent Méditerranée $[9,11]$. Il permet également une meilleure discrétisation de la colonne d'cau.

\subsection{Analyse du bilan}

Des travaux effectués dans des zones plus stables au point de vue hydrologique $[36,39]$ ont mis en évidence des corrélations linéaires entre les quantités intégrées de carbone $(0-100 \mathrm{~m}$ et $0-40 \mathrm{~m})$ et les quantités en sels nutritifs ou en chlorophylle intégrées sur la même couche, ou les concentrations en chlorophylle en surface. Dans notre cas, la relation linéaire (négative) la plus satisfaisante pour décrire les variations de la quantité intégrée de carbone fixé sur la couche $0-100 \mathrm{~m}$ est la profondeur de la couche euphotique [9].

Dans le bilan annuel de carbone proposé ci-dessus, près de $60 \%$ du carbone total ( 80 à $85 \mathrm{~g} \mathrm{~m}^{-2} \mathrm{a}^{-1}$ de C) est fixé au cours de la saison hydrologique hivernale de novembre à mai et $40 \%\left(60\right.$ à $\left.65 \mathrm{~g} \cdot \mathrm{m}^{-2} \cdot \mathrm{a}^{-1} \mathrm{de} \mathrm{C}\right)$ au cours de la saison hydrologique estivale (tableau II). Il faut pourtant moduler cette affirmation puisque l'efflorescence printanière intervient principalement durant la période hydrologique hivernale. Durant cette efflorescence, la production primaire est entre cinq et dix fois plus élevée que durant le reste de l'année.

La répartition du bilan par couche sur la verticale permet de préciser les potentialités d'exportation de matière du système [1,22]. Pendant la saison hydrologique hivernale, ainsi que dans les zones frontales et d'upwelling, la production nouvelle est intense et principalement due à des organismes de grande taille [62]. Les taux de sédjmentation de matière vers les couches profondes sont importants et le zooplancton maintient une forte activité de prédation [44]. Au cours de la saison hydrologique estivale et dans les eaux du large, la production primaire est limitée par les sels nutritifs. Le système est caractérisé par l'abondance de petits organismes et par le stockage de carbone [14] et d'azote [9] sous forme organique dissoute. Cette matière organique dissoute est exportée à la fin de la saison, lors de la rupture de la stratification ; Conan [9] a montré en hiver une bonne relation entre la production nouvelle en surface et la production exportée vers les couches profondes sous forme de particules. $\grave{A}$ l'opposé en été, une fraction importante de la production nouvelle reste piégée dans la couche de surface sous forme de carbone et d'azote organique dissous (COD et NOD). L'exportation d'azote sous forme de NOD représente entre 40 et $90 \%$ de la production nouvelle annuelle totale.

La contribution relative de la couche $50-100 \mathrm{~m}$ au bilan total $(0-100 \mathrm{~m})$ évolue entre 10 et plus de $40 \%$ (figure $6 a$ ). À l'échelle annuelle, la couche $0-50 \mathrm{~m}$ 
contribue pour $75 \%$ au bilan total, contre $25 \%$ pour la couche 50-100 m (tableau II). Le rôle de la couche profonde dans le bilan global est fort à la fin de l'été ( $40 \%$ en septembre), suite à l'enfoncement de la chlorophylle (MPC), mais très irrégulier en l'hiver. La variabilité interannuelle est particulièrement forte comme le prouve l'augmentation significative de 10 à $30 \%$ entre juillet 1993 et 1994 (figure 6a)

Le facteur $\mathrm{f}[20]$ est une mesure de la fertilité du milieu en terme de production exportable [22]. Ce facteur est généralement élevé dans les régions productives et faible en zone oligotrophe. En Méditerranée occidentale, le facteur $f$ varie entre 0,2 et 0,4 [44]. Dans le golfe du Lion, des valeurs de 0,36 [43] ou $0,30-0,37[9,37]$ ont été utilisées. Les fortes valeurs $(>0,7)$ utilisées par Coste et al. [15], Jacques et al. [32], sont largement surestimées selon les revues de Minas et Bonin [43] ou Minas et Codispoti [44].

Avec un facteur $f$ de 0,37 , la production nouvelle dans le golfe du Lion est de 42 à $56 \mathrm{~g} \mathrm{~m}^{-2} \mathrm{a}^{-1}$ de C. Cet intervalle est cohérent avec l'estimation de la production nouvelle potentielle de la zone (46 à $62 \mathrm{~g} \mathrm{~m}^{-2} \mathrm{a}^{-1}$ de C) calculée à partir des quantités de nitrate injectées dans le golfe du Lion par le CNM et le Rhône [13]. À l'échelle annuelle, les deux approches sont opposées, puisque l'une est basée sur des mesures in situ et sur l'utilisation d'un facteur $f$ théorique, alors que l'autre utilise des quantités d'azote disponibles et transformées en carbone à l'aide des rapports de Redfield. Ces approches fournissent des valeurs proches et apportent une certaine validité aux calculs. La totalité du nitrate apportée au niveau du golfe du Lion par les deux principales sources serait donc consommée sur place et le bilan de matière dans la zone concernée serait équilibré.

\section{CONCLUSION}

Dans cette étude, les relations entre le système hydrodynamique et la productivité de la zone sont particulièrement étroites et complexes. Face à la dualité hydrologique saisonnière, il a été possible de décrire trois situations de base du système productif : (1) une situation hivernale ; (2) une situation d'efflorescence à la fin de l'hiver; (3) une situation estivale.

(1) La colonne d'eau est caractérisée par une forte homogénéité et par une variabilité spatio-temporelle importante des mélanges turbulents. La biomasse phyto- planctonique est abondante et productive sur toute la colonne d'eau 0-100 m. Les populations du phytoplancton réagissent et s'adaptent rapidement aux conditions environnementales.

(2) Dans le golfe du Lion, l'efflorescence est précoce (mars-avril) et intervient dans une colonne d'eau peu stratifiée, alors que les flux du CNM sont maximaux. Les quantités de matière exportées par advection horizontale et verticale sont donc significatives.

(3) La situation estivale est caractérisée par un MPC bien défini. La lumière et la diffusion des sels nutritifs sont les facteurs limitant la production.

Les liens fonctionnels entre l'hydrodynamisme et la biomasse phytoplanctonique conduisent à différencier trois zones distinctes de la côte vers le large le long de la radiale prospectée. À l'échelle annuelle, la station M3 apparaît comme intermédiaire car elle subit l'influence alternative de l'eau pauvre du CNM et celle d'eau plus riche provenant de remontées le long de la pente continentale ou d'eau côtière dessalée. La structure frontale sur le bord interne du courant est également un facteur d'enrichissement de la station M3.

Le bilan annuel de la production primaire, à l'entrée du golfe du Lion sur la couche $0-100 \mathrm{~m}$, est de 140 à $150 \mathrm{~g} \mathrm{~m}^{-2} \mathrm{a}^{-1}$ de $\mathrm{C}$, dont près de $75 \%$ sont fixés dans la couche $0-50 \mathrm{~m}$. À l'échelle saisonnière, 80 à $85 \mathrm{~g} \mathrm{~m}^{-2} \mathrm{a}^{-1}$ de $\mathrm{C}$ sont fixés au cours de la saison hydrologique hivernale de novembre à mai, alors que la contribution relative de la couche $50-100 \mathrm{~m}$ varie entre 10 et $40 \%$. Un facteur $\mathrm{f}$ de $0,30-0,37$ conduit à une production nouvelle dans le golfe du Lion de 50 à $56 \mathrm{~g} \mathrm{~m}^{-2} \mathrm{a}^{-1} \mathrm{de} \mathrm{C}$, valeur identique à l'estimation réalisée à partir des flux en nitrate apportés par le Rhône et le CNM [13].

\section{Remerciements}

Ces remerciements s'ajoutent à ceux évoqués dans la première partie de ce travail [13]. Nous tenons à exprimer toute notre gratitude envers notre regretté ami Michel Panouse sans qui le travail de collecte des données n'aurait pu être réalisé. Ces premières missions en sa compagnie ont été très enrichissantes. Nous associons $X$. Durrieu de Madron à cette aide pour la récolte des données darıs la dernière partie du programme. 


\section{RÉFÉRENCES}

[1] Altabet M.A. A time-series study of the vertical structure of nitrogen and particule dynamics in the Sargasso Sea, Limmol. Oceanogr. 34 (1989) 1185-1201.

[2] Altabet M.A., Organic C, N and stable isotopic composition of matter collected on glass-fiber and aluminium oxide filters, Limnol. Oceanogr. 35 (1990) 902-909.

[3] Andersen T., Schartau A.K.L., Paasche E., Quantifying external and internal nitrogen and phosphorus pools, as well ats nitrogen and phosphorus supplied through remineralization, in coastal marine plankton by means of a dilution technique, Mar. Ecol. Prog. Ser. 69 (1991) 67-80.

[4] Banse K., On the dark bottle in the ${ }^{14} \mathrm{C}$ method for measuring marine phytoplankton production, ICES Mar. Sci. Symp. 197 (1993) 132-140.

[5] Berger W.H., Smetacek V.S., Wefer G., Ocean Productivity and Paleoproductivity - An Overview, in : Productivity of the Ocean: Present and Past, Report of the Dahlem Workshop BerIn 1988, John Wiley and Sons. Chichester (1989) 1-34.

[6] Berland B.R., Benzhitsky A.G., Burlakova Z.P., Georgieva L.V., Izmestiev A.M.A., Kholodov V.Y., Maestrini S.Y., Conditions hydrologiques estivales en Méditerranée, répartition du phytoplancton et de la matière organique, in : Océanographie pélagique méditerranéenne, Minas H.J., Nival P. (éd.), Oceanol. Acta 9 (1988) 163179.

[7] Chavez F.P., Buck K.R., Bidigare R.R., Karl D.M., Hebel D.. Latasa M., Campbell L., On the chlorophyll a retention properties of glass-fiber GF/F filters, Limnol. Oceanogr. 40 (2) (1995) 428-433.

[81 Christaki U., Van Vambeke F, Epaminondas C., Conan P., Gaudy R., Food web structure variability in the surface layer at a fixed station influenced by the North Western Mediterranean Current, Hydrobiol. 321 (2) (1995) 145-153.

[9] Conan P., Variabilité et bilan de fa production primaire en zone côtière (Méditerranée nord-occidentale, entrée du golfe du Lion) en relation avec les systèmes biologique, chimique et hydrodynamique (courant nord méditerranéen), thèse doct. univ. Méditerranée. Aix-Marseille-II (1996) $290 \mathrm{p}$.

[10] Conan P. Millot C., Variability of the Northern Current in the Western Mediterranean Sea from February to June 1992, Uceanol. Acta 18 ( I995) ]93-205.

[11] Conan P., Pujo-Pay M., Estimation of primary production ( ${ }^{11} \mathrm{C}$ ) in the Gulf of Lions during July 1993. Improvement of a new apparatus for assessing primary production, Wa1. Poll. Res. Rep., EROS 2000, $5^{\circ}$ workshop 28-30 mars 1994, Martin J.M., Barth H. (éd.) (1994) 77-8?.

[12] Conan P., Pujo-Pay M., Leveau M. Raimbault P.. Une autre utilisation du système Let-Go : échantillonnage rapide d'une colonne d'eau entre 0 et $200 \mathrm{~m}$, Ann. Inst. Océanogr. 72 (2) (1996) 221-227.

[13] Conan P., Pujo-Pay M.. Raimbault P., Leveau M., Variabilité hydrologique et biologique du galfe du Lion, I. Transports en azote et productivité potentielle, Oceanol. Acta 21 (6) (1998) $751-765$.

[14] Copin-Montégut G., Avril B., Vertical distribution and temporal variation of dissolved organic carbon in the North-Western Mediterranean Sea. Deep-Sea Res. 40 (1994) 1963-1972.

[15] Coste B.. Gostan J., Minas H.J., Influence des conditions hivernales sur les productions phyto et zooplanctoniques en Méditerranée nord-occidentile, I. Structures hydrologiques et distribution des sels nutritifs, Mar. Biol. 16 (1972) 320-348.

[16] Dandonneau Y., Measumenent of in silu profiles of primary production using an automated sampling and incubation device. ICES Mar. Sci. Symp. 197 (1993) 173-180.

[17] Dandonneau Y., Le Bouteiller A., A simple and rapid device for measuring planktonic primary production by in situ sampling, and $\mathrm{Cl} 4$ injection and incubation. Deep-Sea Res. 39 (1992) 795-803.

[18] Dore J.E. Karl D.M.. Nitrification in the euphotic zone as a source for nitrite, nitrate, and nitrous oxide at Station Aloha, Limnol. Oceanogr. 41 (1996) 1619-1628.

[19] Dring M.J., Jewson D.H., What does ${ }^{14} \mathrm{C}$ uptake by phytoplankton really measure? A theoretical modelling approach, Proc. R. Soc. Lond. B 214 (1982) 351-368.

[20] Dugdale R.C., Goering J.J.. Uptake of new and regenerated forms of nitrogen in primary productivity, Limnol. Oceanogr. $12(1967) 196-206$

[21] Durrieu de Madron X.. Panouse M., Transport de matière particulaire en suspension sur le plateau continental du golfe du Lion. Situations estivales et hivernales, C.R. Acad. Sci. 322 (IIa) (1996) 106]-1070.

[22] Eppley R.W, Peterson B.J., Particulate organic matter and planktonic new production in deep ocean, Nature 282 (1979) $311-323$

[23] Eppley R.W., Sharp J.F., Photosynthetic measurements in the central North Pacific : dark loss of carbon in $24 \mathrm{~h}$ incubations, Limnol. Oceanogr. 20 (1975) 981-987.

[24] Estrada M., Primary production at the deep chlorophyll maximum in the western Mediterranean, Proceedings of the 19th European Marine Biology Simposium, Gibbs P.E. (éd.), Cambridge University Press (1985) 109-121.

[25] Fitzwater S.E., Kuauer G.A., Murtin J.M., Metal contamination and its effect on primary production measurements, Limnol. Oceanogr. 27 (1982) 544-551.

[26] Glibert P., Conley D.J., Fisher T.R., Harding Jr. L.W., Malone T.C. Dynamics of the 1990 winter/spring hloom in Chesapeake Bay, Mar. Ecol. Prog. Ser. 122 (1995) 27-43.

127| Herbland A.. The deep phaeopigment maximum in the ocean: Reality or illusion? In : Towards a serie on biological-physical interaction in the world ocean, Rotschild (éd.), Kluver Ac. Publishers (1987) 157-172. 
[28] Herbland A., Le Borgne R., Voituriez. B., Structure hydrologique et production planctonique dans l'Atlantique tropical oriental, thèse doct. État, univ. d'Aix-Marseille-II (1983) 98 p.

[29] Herbland A., Le Bouteiller A, Raimbault P., Size structure of phytoplankton in the Equatorial Atlantic Ocean. Deep-Sea Res. 32 (1985) 819-836.

[30] Jacques G., Aspects quantitatifs du phytoplancton de la région de Banyuls-sur-mer, (golfe du Lion) 1965-1969, thèse doct. Sci. nat., Fac. sci. Paris (1970) 210 p.

[31] Jacques G., Flux de carbone en milieu pélagique de Méditerranée occidentale lors de la floraison printanière, in : Océanographie pélagique méditerranéenne, Minas H.J., Nival P. (éd.). Oceanol. Acta 9 (1988) 143-148.

[32] Jacques G., Cahet G., Fiala M., Panouse M., Enrichissement de communautés phytoplanctoniques néritiques de Méditerranée nord-occidentale, J. exp. mar. biol. Ecol. 11 (1973a) 287295.

[33] Jacques G., Minas H.J., Minas M., Nival P., Influence des conditions hivernales sur les productions phyto et zooplanctoniques en Méditerranée nord-occidentale, II. Biomasse et production planctonique, Mar. Biol. 23 (1973b) 251-265.

[34] Klein P., Coste B., Effects of winds stress variability on nutrient transport into the mixed layer, Deep-Sea Res. 31 (1984) $21-27$.

[35] Lacombe H., Tchernia P., Caractères hydrologiques et circulation des eaux en Méditerranée, in : The Mediterranean Sea. Stanley D.J. (éd.), Dowden Hutchinson Ross Stroudsburg, PA (1972) 25-36.

[36] Le Bouteiller A., Herbland A., Carbon fixation and productivity index in relation to chlorophyll and light in the equatorial Atlantic ocean, Océanogr. trop. 19 (1984) 161-179.

[37] Lefèvre D., Minas H.J., Minas M., Robinson C. Williams P.J.Le B.. Woodward E.M.S., Review of gross community production, primary production, net community production and dark respiration in the gulf of Lions, Deep-Sea Res. (1997) 44 $801 \div 832$.

[38] Lévy M., Mémery L., André J.M., Simulation of primary production and export fluxes in the Northwestern Mediterranean Sea, J. Mar. Res. 56 (1998) 197-238.

[39] Lohrenz S.E., Wiesenburg D.A., De Palma I.P., Johnson K.S., Gustafson D.E.. Interrelationships among primary production, chlorophyll, and environmental conditions in frontal regions of the western Mediterranean Sea, Deep Sea Res. 35 (1988) 793 810 .

[40] Millot C.. Circulation in the western Mediterranean Sea, Oceanol. Acta 10 (1987) 143-149.

[41] Millot $C$., The Gulf of Lions' hydrodynamics, Cont. Shelf Res. $10(1990) 885-894$.

[42] Minas H.J.. Recherches sur la production organique primaire dans le bassin méditerranéen nord-occidental. Rapports avec les phénomènes hydrologiques, thèse doct. État, univ. AixMarseille-II. (1968) $228 \mathrm{p}$.

[43] Minas H. J., Bonin M.C., Oxygénation physique et biologique de la Méditerranée nord-occidentale en hiver et au printemps. in : Océanographie pélagique méditerranéenne, Minas H.J., Nival P. (éd.), Oceanol. Acta 9 (1988) 123-132.

[44] Minas H.J., Codispoti L.A., Estimation of primary production by observation of changes in the mesoscale nitrate field, ICES mar. Sci. Symp. 197 (1993) 215-235.

[45] Minas H. J., Minas M., Coste B., Gostan J., Nival P., Bonin M.C., Production de base et de recyclage : une revue de la problématique en Méditerranée nord-occidentale, in : Océanographie pélagique méditerranéenne, $H$. J. Minas et $P$. Nival (éd.) Oceanol. Acta 9 (1988) 155-162.

[46] Mingelbier M., Klein B., Claereboudt M.R., Legendre L., Measurement of daily primary production using $24 \mathrm{~h}$ incubations with the ${ }^{14} \mathrm{C}$ method: a caveat, Mar. Ecol. Prog. Ser. 113 (1994) 301-309.

[47] Morel A., Light and marine photosynthesis: a spectral mode] with geochemical implications, Prog. Oceanog. 26 (1991) $263-306$.

[48] Morel A., André J.M., Pigment distribution and primary production in the Western Mediterranean as derived and modeled from coastal zone scanner observation, J. Geophys. Res. 96 (C7) (1991) $12685-12698$.

[49] Parsons T.R.. Takahashi M., Hargave B., Biological Oceanic Processes ( $3^{\mathfrak{E}}$ éd.). Pergamon Press, Angleterre, 1984, $300 \mathrm{p}$.

[50] Peterson B.J., Aquatic primary productivity and the ${ }^{14} \mathrm{C}-\mathrm{CO} 2$ method: A history of the productivity problem. Ann. Rev. Ecol. Syst. 11 (1980) 259-385.

[51] Pujo-Pay M., L'azote et le phosphore organique et minéral en milieu marin, thèse doct. univ. Méditerranée, Aix-Marseille-II (1995) $200 \mathrm{p}$.

[52] Pujo-Pay M., Conan P., Raimbault P.. Particulate and dissolved organic nitrogen and phosphorus in the northwestern Mediterranean Sea (Eros 2000-Discovery cruise 1993), Wat. Poll. Res. Rep. EROS $2000,5^{\circ}$ workshop 28-30 mars 1994 Martin J.M., Barth H. (éd.) (1994) 79-86

[53] Pujo-Pay M., Raimbault P., Conan P., Sous-estimation des teneurs en azote particulaire en milieu océanique par l'utilisation des filtres GF/F, C. R. Acad. Sc. 324 (Ila) (1997) 40I-407.

[54] Raimbault P., Taupier-Letage 1., Rodier M., Vertical distribution of phytoplankton in the western Mediterranean Sea during early summer, Mar. Ecol. Prog. Ser. 45 (1988) 153-158.

[55] Rodhe W., The primary production in lakes : some results and restrictions of the ${ }^{14} \mathrm{C}$ method, Rapp. P.V. Réun. Cons. Perm. Int. Explor. Mer 144 (1958) 122-128.

[56] Rowe G.T., Smith S., Falkowsky D., Withledge T., Theroux R., Phoel W., Ducklow H., Do continental shelves export organic matter? Nature 324 (1986) 544-561.

[57] Slawyk G., Raimbault P., A simple procedure for the simultaneous recovery of dissolved inorganic and organic nitrogen in ${ }^{15} \mathrm{~N}$-tracer experiments on oceanic waters improving the isotopic mass balance, Mar. Ecol. Prog. Ser, 124 (1995) 289-299.

[58] Smith S.V., Hollibaugh J.T. Coastal metabolism and the oceanic organic carbon balance, Rev. Geophys. 31 (1) (1993) 75 89. 
[59] Sournia A., Brylinsky J.M., Dallot S., Le Corre P., Leveau M., Prieur L., Froget C., Fronts hydrologiques au large des côtes francaises : les sites-ateliers du programme frontal, Oceanol. Acta 13 (1990) 413-438.

[60] Steemann-Nielsen E., Measurement of the production of the organic matter in the sea by mean of carbon 14, Nature (195I) $167684-685$.

[61] Taupier-Letage I., Millot C., General hydrodynamical features in the Ligurian Sea inferred from the Dyomé experiment, in : Océanographie pélagique méditerranéenne, Minas H.J., Nival P. (Eds.) Oceanol. Acta 9 (1986) 119-131.

[62] Thingstad T.F., Rassoulzadegan F., Nutrient limitations, microbial food webs, and biological C-pumps: suggested interactions in a P-limited Mediterranean, Mar. Ecol. Prog. Ser. 117 (1995) 299-306

1631 Townsen D.K., Keller M.D., Sieracki M.E., Ackleson S.G., Spring phytoplankton blooms in the absence of vertical water stratification, Nature 360 (1992) 59-62. $\lceil 64\rceil$ Travers A., Travers M., Données sur quelques facteurs de l'écologie du plancton dans la région de Marseille. 2. La température, les précipitations, la stabilité et la circulation des eaux, Téthys 4 (1972) 515-534.

[65] Verity P.G., Smetacek V., Organism life cycles, predation and marine pelagic ecosystems, Mar. Ecol. Prog. Ser. 130 (1996) 277-293.

[66] Videau C., Leveau M., Biomasse et production phytoplanctonique dans le front du panache rhodanien. Situation printanière, C. R. Acad. Sci. 3Il (III) (1990) 219224.

[67] Williams P.I.LeB., Chemical tracer methods for measuring plankton production, ICES Mar. Sci. Symp. 197 (1993) 20-36.

[68] Williams P.J.LeB., Lefèvre D., Algal ${ }^{14} \mathrm{C}$ and total carbon metabolisms. 1. Models to account for respiration recycling, J. Plankt. Res. 18 (10) (1996) 1941-1959.

[69] Zakardjian B., Role du système advection/diffusion sur la production primaire en zone frontale, thèse doct., univ. P.M.-Curie Paris-VI (1994) 325 p. 